University of Nebraska - Lincoln

DigitalCommons@University of Nebraska - Lincoln

Faculty Publications in Food Science and Technology

Food Science and Technology Department

2014

\title{
Translating reference doses into allergen management practice: Challenges for stakeholders
}

René W. R. Crevel

Joseph Baumert

Stefano Luccioli

Athanasia Baka

Sue Hattersley

See next page for additional authors

Follow this and additional works at: https://digitalcommons.unl.edu/foodsciefacpub

Part of the Allergy and Immunology Commons, Food Science Commons, and the Public Health Commons

This Article is brought to you for free and open access by the Food Science and Technology Department at DigitalCommons@University of Nebraska - Lincoln. It has been accepted for inclusion in Faculty Publications in Food Science and Technology by an authorized administrator of DigitalCommons@University of Nebraska Lincoln. 
Authors

René W. R. Crevel, Joseph Baumert, Stefano Luccioli, Athanasia Baka, Sue Hattersley, Jonathan O.B. Hourihane, Stefan Ronsmans, Frans Timmermans, Rachel Ward, and Yong-joo Chung 


\title{
Invited Review
}

\section{Translating reference doses into allergen management practice: Challenges for stakeholders}

\author{
René W.R. Crevel ${ }^{\mathrm{a}}$, Joseph L. Baumert ${ }^{\mathrm{b}}$, Stefano Luccioli ${ }^{\mathrm{c}}$, Athanasia Baka ${ }^{\mathrm{d}, *}$, Sue Hattersley ${ }^{\mathrm{e}}$, \\ Jonathan O'B. Hourihane ${ }^{\mathrm{f}}$, Stefan Ronsmans ${ }^{\mathrm{g}}$, Frans Timmermans ${ }^{\mathrm{h}}$, Rachel Ward ${ }^{\mathrm{i}}$, Yong-joo Chung ${ }^{\mathrm{j}}$ \\ a Safety and Environmental Assurance Centre, Unilever, Colworth House, Sharnbrook, Bedford, UK \\ ${ }^{\mathrm{b}}$ Food Allergy Research and Resource Program, University of Nebraska, Lincoln, USA \\ ${ }^{c}$ Center for Food Safety and Applied Nutrition, FDA, USA \\ ${ }^{\mathrm{d}}$ International Life Sciences Institute-ILSI Europe, Brussels, Belgium \\ ${ }^{\mathrm{e}} U K \mathrm{~K}$ Food Standards Agency, London, UK \\ ${ }_{\mathrm{f}}^{\mathrm{f}}$ Department of Paediatrics, School of Medicine, University College Cork, Cork, Ireland \\ ${ }^{g}$ Coca-Cola Services, Brussels, Belgium \\ ${ }^{\mathrm{h}}$ European Anaphylaxis Taskforce and Nederlands Anafylaxis Netwerk, Dordrecht, The Netherlands \\ ${ }^{\mathrm{i}} \mathrm{R}$. Ward Consultancy Limited, Nottingham, UK \\ ${ }^{\mathrm{j}}$ Nestlé Research Centre, Lausanne, Switzerland
}

\section{A R T I C L E I N F O}

\section{Article history:}

Received 5 August 2013

Accepted 20 January 2014

Available online 31 January 2014

Keywords:

Food allergy

Public health

Risk assessment

Probabilistic modelling

Eliciting dose

Reference dose

\begin{abstract}
A B S T R A C T
Risk assessment describes the impact of a particular hazard as a function of dose and exposure. It forms the foundation of risk management and contributes to the overall decision-making process, but is not its endpoint. This paper outlines a risk analysis framework to underpin decision-making in the area of allergen cross-contact. Specifically, it identifies challenges relevant to each component of the risk analysis: risk assessment (data gaps and output interpretation); risk management (clear and realistic objectives); and risk communication (clear articulation of risk and benefit). Translation of the outputs from risk assessment models into risk management measures must be informed by a clear understanding of the model outputs and their limitations. This will lead to feasible and achievable risk management objectives, grounded in a level of risk accepted by the different stakeholders, thereby avoiding potential unintended detrimental consequences. Clear, consistent and trustworthy communications actively involving all stakeholders underpin these objectives. The conclusions, integrating the perspectives of different stakeholders, offer a vision where clear, science-based benchmarks form the basis of allergen management and labelling, cutting through the current confusion and uncertainty. Finally, the paper recognises that the proposed framework must be adaptable to new and emerging evidence.
\end{abstract}

(C) 2014 ILSI Europe. Published by Elsevier Ltd. All rights reserved.

\section{Contents}

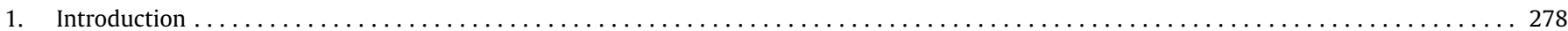

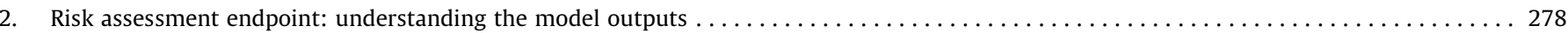

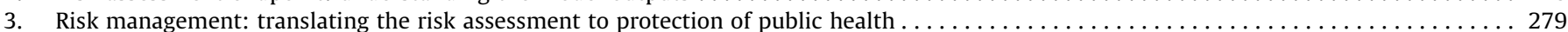

3.1. Scope: risk management objectives in relation to quantitative risk assessment related to cross-contact ............... 279

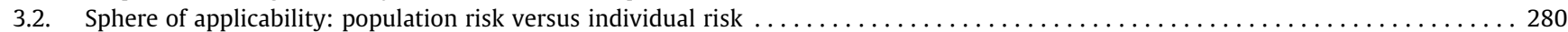

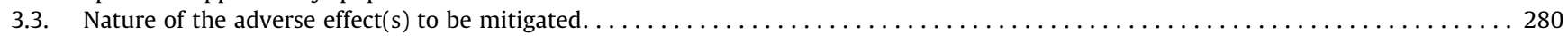

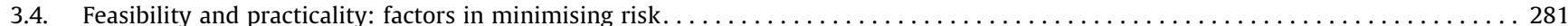

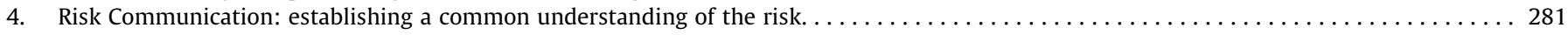

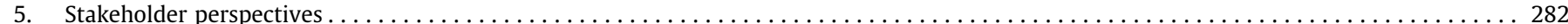

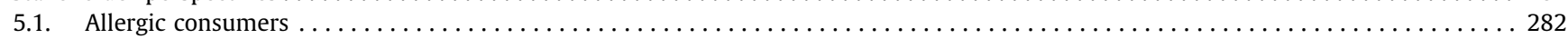

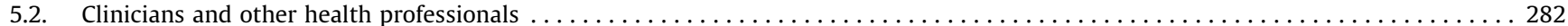

\footnotetext{
* Corresponding author. Address: ILSI Europe a.i.s.b.l., Avenue E. Mounier 83, Box 6, 1200 Brussels, Belgium. Tel./fax: +32 27620044

E-mail address: publications@ilsieurope.be
} 


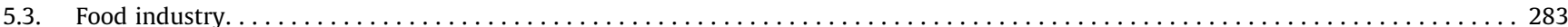

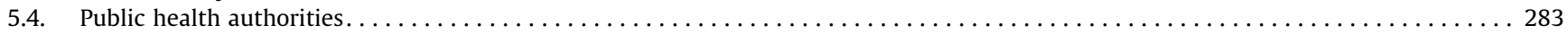

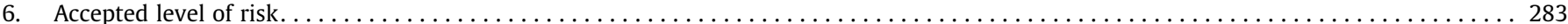

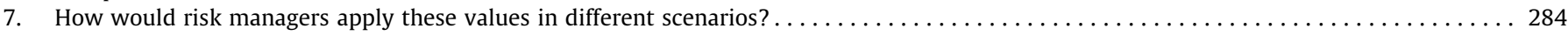

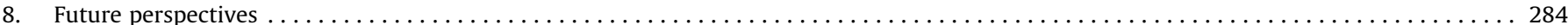

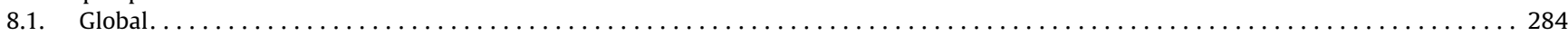

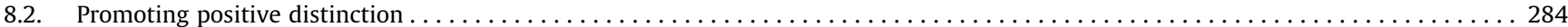

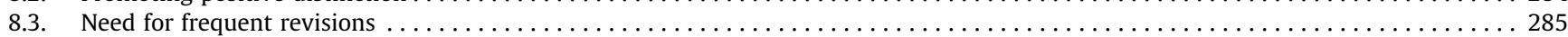

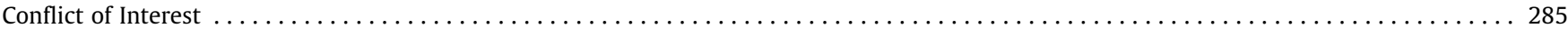

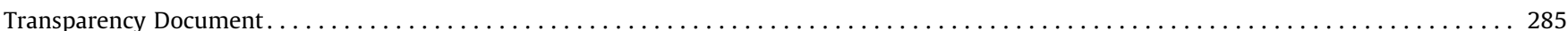

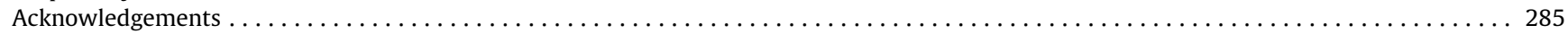

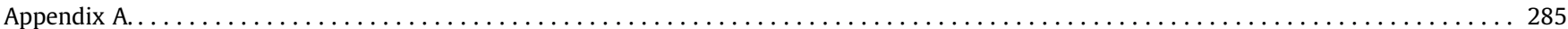

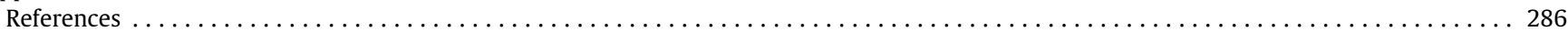

\section{Introduction}

In the previous papers, we demonstrated that the risk from unintended food allergens can be assessed and quantified using the same approaches as for other contaminants. However, risk assessment sets out the (health) consequences of exposure, but does not indicate whether the risk in question is acceptable or not, or at what point it would become so. It also does not deal with the challenges of implementation. This paper discusses the elements that need to be considered in arriving at such a conclusion. It starts by examining the meaning of the risk assessment endpoint generated by the methodologies previously described in the risk assessment paper and how these might be communicated to the different stakeholders. It then considers the implications, benefits and potential pitfalls for each group of adopting specific reference values, in particular those proposed for the Allergen Bureau of Australia and New Zealand's Voluntary Incidental Trace Allergen Labelling (VITAL) system. Finally, it offers a vision for allergen management as it moves forward. The framework discussed is intended to be applied to manage the risk from allergen crosscontact in foods for normal consumption. It is not intended to provide the basis for claiming that a product is "free-from" a specific allergen, although it may help in deriving criteria for doing so.

\section{Risk assessment endpoint: understanding the model outputs}

Risk assessment is the process whereby the impact of a hazard is estimated, and is a critical prerequisite to management of the risk. However, deciding on appropriate risk management measures requires that stakeholders agree on what degree of risk they accept. Both the frequency of adverse effects and the nature of those effects in terms of severity, duration and reversibility will determine acceptability (or perhaps more correctly tolerability). Severe and irreversible effects will thus be tolerated to a lower extent than those with a lesser impact on health, as will those whose consequences are seen as unpredictable (Madsen et al., 2012). The models described in the previous paper (ref) predict the potential proportion of allergic people reacting to a range of doses of the specific allergen (dose distributions) or the total number of reactions expected if a given distribution of allergen is present in a food product of interest. However, this information may not clearly address the potential of these allergens to cause serious or irreversible effects on health, which drives much of the public health (as well as individual) concern on the need for current allergen labelling and strict avoidance policies (Madsen et al., 2010, 2012). Thus, the likely severity of a reaction at any given dose is also critical to the risk assessment and consequent risk management measures. The scarcity of data on the relationship between dose and severity therefore remains an important gap in characterising the risk from exposure to food allergens. Furthermore, a common understanding among stakeholders about the endpoint(s) which the risk assessment seeks to evaluate and quantify is needed to achieve transparency and eventual acceptance of the risk management strategy.

To date only limited information has emerged regarding the relationship between food challenge data and clinical reactions in daily life. During low dose challenge, patients are closely monitored and once a reaction has clearly taken place (usually if objective symptoms occur) the challenge is stopped and the patient treated (if necessary). Consequently the reaction during challenge will usually be not as severe as reactions can be in daily life. There is also no clear relationship between minimum eliciting dose and severity (Rolinck-Werninghaus et al., 2012). However one can assume that if threshold data are derived from a representative population these factors will minimally influence the threshold distribution itself. To further illustrate the health implications of findings from threshold distributions, challenge studies have been proposed to evaluate whether the dose corresponding to the ED05 indeed leads to responsiveness in $5 \%$ of the patients and to provide information on the severity profile of the reactions observed (Zurzolo et al., 2013) Theoretically reactions during DBPCFC can be influenced by preceding doses in the dose escalation, a problem which is avoided by challenge with a single dose. In real life, thresholds and subsequent reactions may also be influenced by a number of known and unknown factors, such as exercise, infection, stress and more. Quantification of the potential effects, if any, of these factors will require further research.

Allergic reactions can range in severity from barely perceptible subjective symptoms, such as itching or tingling in the mouth, to mild to moderate objective symptoms, such as hives, lip swelling, nasal congestion, diarrhea or vomiting, to severe breathing difficulties and life-threatening anaphylactic shock. Clearly the implications of these different types of reactions from both an individual as well as a public health perspective differ greatly and need to be taken into account in risk assessments. In the USA, the Food Allergen Labeling and Consumer Protection Act (FALCPA) refers to the labelling exemption concept of an "allergic response that poses a risk to human health" as what management of the risk seeks to avoid. This terminology implies that there are some allergic responses which can be deemed not to pose a risk to human health. This also accords with the observation that the concept of risk itself according to many definitions, as well as in the public mind, is associated not only with the probability of an adverse effect, but also with consideration of its likely severity.

One of the difficulties in applying this concept in food allergen risk assessment has been the perception that severity is unpredictable, such that a history of non-severe reactions is no guarantee that future reactions will not be more severe. Extrinsic factors, such as exercise and medication can undoubtedly modulate severity, as discussed already. However, when evaluating conclusions that severity is unpredictable, due regard must be paid to the fact that those conclusions are often based on circumstances where no information exists on a very important modulator of effect, namely dose. 
Current models for assessing the risk from the unintended presence of allergenic constituents through cross-contact focus solely on a calculation of the probability of any reaction (or any objective reaction) without any regard to its health impact. This reflects the available data. However, even a fairly cursory examination of the outputs of these models reveals that the number of predicted reactions far exceeds the numbers that are recorded. Thus a fairly approximate calculation based on the paper of Rimbaud and colleagues (Rimbaud et al., 2010) predicts about 65,000 reactions to peanut in France from chocolate alone, which is 2-4 times higher than the number of severe allergic reactions from all combined food allergens estimated by Rance et al. (2005). This may not indicate solely inevitable inaccuracies and uncertainties in the risk assessment model, but could reflect, among other factors, that a large proportion of reactions at low doses have such limited impact on health that they are, and will always remain invisible to any monitoring or recording system. To put the issue of contamination in perspective, in a small study on the relationship between dose of peanut and the occurrence of anaphylaxis in an experimental setting, Wainstein and colleagues (Wainstein et al., 2010) showed that the minimum dose associated with an anaphylactic reaction was the equivalent of $5 \mathrm{mg}$ of peanut protein ( $20 \mathrm{mg}$ peanut). This is 25 -fold greater than the mean unintended peanut intake per occasion per week estimated by Rimbaud and colleagues $(0.2 \mathrm{mg})$ and the proposed VITAL reference dose for peanut (Allergen Bureau, 2012), (Allen et al., 2013; Taylor et al., 2014).

In considering whether uncertainty factors need to be applied to reference doses derived from dose distributions, it is useful to remember that the model predictions for any given dose represent the total number of reactions, of which a relatively high proportion may be expected to be mild or inconsequential. If the risk assessment goal sought through the application of reference doses (e.g. in VITAL) is to minimise the number of reactions which would be considered harmful to human health, then one could argue that the models themselves incorporate their own inbuilt uncertainty factor. Notwithstanding this observation, more knowledge on the relationship between dose and severity would help to improve risk assessments and agreement on an accepted level of risk. Food challenge data provide relatively limited, but nevertheless useful information in this regard and analysis of the Europrevall dataset has examined ways to use it in risk assessments.

Another, complementary, approach would be to use the Delphi technique to generate a consensus among clinical experts about the likelihood of a severe reaction and the relative severity of the associated responses for an appropriate range of very low doses, such as those proposed by the VITAL Scientific Panel and adopted by this Expert Group. These probability and severity elements could then be incorporated into probabilistic risk assessment models.

How a risk is expressed can also influence how it is perceived. However, a critical first step is to ensure that whatever form of expression is used is accurate. Typically, the result of a risk assessment is expressed as the likelihood of a reaction (e.g. 1\%, 1 in a million, etc.). However, a risk assessment includes intake data (even if inadequate) and such data are expressed as intake over a specified period of time. Thus the resulting expression of risk at the very least should indicate the time denominator, or where appropriate the number of predicted reactions per unit of product and, in ideal terms, reactions per estimated meal consumption of product.

Beyond this, careful use of terminology is necessary to reflect the quantitative models. For instance, a reference dose (ED01) can be described equally accurately as the dose equivalent to a 1 in 100 chance of a reaction or the dose which offers $99 \%$ protection to the allergic population. Results can also be expressed in a variety of ways for an individual, such as the number of years this individual could be expected to live without experiencing a reaction. One possible way forward would be to anchor those risk determinations by reference to other risks of a similar nature (Madsen et al., 2010).

\section{Risk management: translating the risk assessment to protection of public health}

Risk management can only take place effectively if its aims are clearly set out and understood. A clear description of risk management objectives should therefore precede any risk assessment as it will guide the collection of the appropriate data. In particular, the potential constraints on these objectives need to be identified and addressed. Critical considerations include:

- Feasibility within operational and regulatory constraints: it is important to ensure that any proposed levels do not have the unintended consequence of making the situation worse. A highly conservative approach, if it resulted in reference doses that were beyond the technological capabilities of most food manufacturing facilities, could result in an increase in precautionary labelling, at least in the short to medium term. Indeed, this feasibility issue was one of the concerns acknowledged by the US FDA in its assessment of gluten thresholds or action levels for gluten-free label rulemaking [Federal Register of August 5, 2013 ( 78 FR 47154)]. The complexity involved in achieving these objectives therefore needs to be discussed with all stakeholders.

- Need to balance a number of potentially conflicting considerations:

- Potential effects on other safety parameters (microbiological, chemical).

- Potential adverse environmental aspects of e.g. cleaning protocols.

- Potential wastage of otherwise safe food product.

- Potential over-use of precautionary statements - If a precautionary warning is used, how well do allergic consumers adhere to it?

- Decreased quality of life for allergic consumers due to constrained food choices from over-use of precautionary statements and, conversely, due to increased fear and anxiety of reactions to products without such statements. Also, constrained food choices may lead some individuals to begin disregarding precautionary labels and lead to ill-advised risk-taking behaviour.

More generally, defining risk management objectives includes clarity about the scope of the risk management activity, what it applies to (and equally importantly, what it does not apply to) as well as what type of adverse events it seeks to mitigate, among the range of reactions that a condition like food allergy can produce.

\subsection{Scope: risk management objectives in relation to quantitative risk} assessment related to cross-contact

Management of allergen risks encompasses a wide range of activities, including setting of ingredient specifications, verification of label artwork, checks on packaging materials, production planning, etc. Specific management of cross-contact risks is only one of those elements. Risk as described and discussed in this series of reports focusses on the risk posed by the unintended presence of allergens through cross-contact at any point in the supply chain. It therefore excludes the risk arising from other activities within allergen risk management, for instance, undeclared allergen as a result of mislabelling or mispackaging.

Quantitative risk assessment using reference doses aims principally at minimising the risk from the presence of unintended allergen. However, it could also inform decisions regarding mandatory 
ingredient labelling regulations, including exemptions from allergen labelling requirements for highly processed ingredients from allergenic foods that have been demonstrated not to pose an allergenic risk. Food labelling regulations in most countries mandate the source labelling of all ingredients derived from or containing protein from commonly allergenic foods, unless exempted. Ingredients currently exempted from source labelling include those exempted either by the prevailing food law (e.g. highly refined oils in the US) or by a specific exemption process (several ingredients in the EU) and represent a very small number of ingredients. Many ingredients labelled as allergens on the market contain very high levels of protein from the allergenic source (e.g. casein from milk or semolina flour from wheat) and represent a clear risk to allergic individuals. However, others contain either very low (e.g. soy lecithin or food-grade lactose from milk) or no detectable protein from the allergenic source based on the limits of current detection methods (e.g. milk-based flavourings such as butter ester). More importantly, these latter ingredients remain labelled and avoided by consumers even though there is little to no evidence that residual allergenic protein from such ingredients would pose a clinically significant risk to public health. Thus, required source labelling of ingredients, in cases where very little allergenic protein remain and residual risk is extremely low, may serve to further restrict the diets of food-allergic consumers with little or no public health benefits.

Up to now, decisions regarding the necessity of source labelling for ingredients with very low levels of protein from the allergenic source have not been based upon quantitative risk assessment. However, since the allergen concentrations of ingredients as well as the amount(s) of the same ingredients added to the product are both known with reasonable precision, allergen content in this type of product labelling scenario is likely easier to estimate and control by the food industry compared to scenarios involving unintended allergens. Thus, it is conceivable that an accurate risk assessment of allergen levels in the final product from these ingredient scenarios can be achieved, and this information can ultimately inform risk-based decisions on the necessity of source labelling for specific food ingredients as well.

\subsection{Sphere of applicability: population risk versus individual risk}

The allergen risk assessment approach outlined above addresses allergenic risk at the population level, aiming to provide a defined level of protection. This approach does not, and indeed cannot, indicate what risk would be incurred by any specific individual under specific circumstances. In this regard, it cannot guarantee that every individual would be protected, but can only provide a probability estimate. This approach, therefore, is not dissimilar from chemical risk assessment, in which the accepted risk is defined in terms of any additional case of adverse health effect (e.g. cancer) over a lifetime without information about which individual(s) will be the additional case(s). While bearing in mind the limitations above, information from the risk assessment can be used in conjunction with other information (e.g. clinical knowledge of their minimum eliciting dose and pattern of reactivity) specific to the person concerned to provide helpful information about the risks incurred by that person. As discussed earlier, determining what is safe (what risk is accepted for individual allergic consumers) using a population approach is a shared responsibility between the different stakeholders.

\subsection{Nature of the adverse effect(s) to be mitigated}

Insofar as preventing serious and irreversible adverse effects is more important than avoiding transient mild ones, the risk management objectives must take into account the nature of the adverse effect(s) that are likely to occur at the limits (e.g. reference doses) used to guide risk management actions. Informed stakeholders in the field of food allergy now accept that total elimination of the risk of reaction from incidental exposure to allergen is not feasible (Madsen et al., 2012). As discussed previously, the manifestations of food allergy range from barely perceptible symptoms to severe anaphylaxis, which can lead to death. Thus the principal risk management objective could be to improve food safety for food allergic consumers by preventing severe or life-threatening reactions from unintended (not undeclared) presence of allergen, while minimising risk for other types of reactions to manageable levels. A clear description of the risk management objectives will also help subsequent risk communication. Once the principal risk management objective has been agreed, subsidiary objectives can be defined which contribute to the realisation of this principal objective. One such objective could be for industry to harmonise the application of precautionary labelling and make it more transparent to other stakeholders. This would increase the understanding of its meaning and thereby enhance its credibility and decrease risk taking among allergic consumers. The reference doses defined by dose distribution modelling, as well as the outputs of probabilistic risk assessment need to be interpreted in the light of those risk management objectives. With regards to nature of the adverse effect, in particular, it is important to remember that those outputs represent numbers of reactions but do not indicate how many of those reactions might be severe.

A particular consideration for the risk management objectives will be achieving the appropriate balance in terms of the proportion of products bearing a precautionary label. Precautionary 'may contain' labelling is applied to a significant number of food products in certain categories (Pieretti et al., 2009) and clinical guidelines conservatively recommend that all patients with food allergies avoid all products with this type of labelling (Boyce et al., 2010). Since this labelling is often applied to products which present minimal risks arising from the unintended presence of the allergenic food, one of the consequences of this labelling is that it significantly restricts the choice of potentially safe foods available to allergic consumers. This food choice restriction not only negatively impacts the quality of life of these individuals but increases the risk of a nutritionally deficient diet for food allergic consumers as well (Christie et al., 2002). Importantly, as evidenced by a sizable and growing percentage of consumers who report distrust and general disregard for precautionary label statements, with some types of statements (e.g., "produced in a facility") likely to be disregarded over others (e.g. "may contain") (Barnett et al., 2011a; Hefle et al., 2007; Leung et al., 2003b, 2003a), the large number of products carrying precautionary labelling detracts from the value of the warning in those cases where it is appropriate. As a general principle, precautionary labelling should only be used after a thorough risk assessment and where actions intended to minimise the likelihood of allergen cross contamination have not been able to reduce the risk sufficiently. Over the last decade, much progress has been made in filling a number of the data and knowledge gaps that prevented an adequate assessment of the risk, as detailed earlier in this report.

These developments lead to a realistic possibility that a solid and consistent, science-based framework for dealing with specific food incidents (product non-conformance with potential safety implications) and for managing the risks posed by various scenarios involving allergen cross contamination can also be achieved. Such a solid framework also ensures that a consistent risk-based compliance and enforcement approach can be adopted, and helps to maintain a proportionate response to different incidents within a country. Above all, a broadly similar approach might be envisaged which is consistent between countries across the EU and beyond. By fostering development of the new knowledge on thresholds and its translation into an innovative risk assessment concept, businesses can 
develop and apply a comprehensive and self-regulatory approach which encompasses labelling of allergenic food used deliberately as ingredients, risk-based allergen cross contamination warnings and also allergy 'free from' claims. Such developments offer the possibility of risk management objectives, based on sound evidence and scientific understanding, with a quantitative basis where applicable.

\subsection{Feasibility and practicality: factors in minimising risk}

Determining what might be a tolerable risk needs to balance the level of residual risk against the benefits to the allergic consumer of having a transparent, science-based framework that allows more confidence and trust in the precautionary labelling being provided. If the action level/threshold is set as low as possible, this will appear to provide greater protection. However, setting a very low level could mean that businesses might not be able to work to such a level and still produce food products at realistic cost. It could also result in many more food products having precautionary labelling, thereby severely curtailing the choice of foods. The framework therefore needs to be both feasible and practicable. If it does not meet those conditions, then adoption will be reluctant and take-up by industry will be poor. Moreover, the framework will fail to meet the objectives it was designed to address or, even worse, might lead to unintended consequences opposite to those desired. One example of this latter point might be an extension of precautionary labelling, rather than a reduction or stabilisation. This could lead to an increase in potential direct or indirect adverse health consequences from not only fewer food choices for allergic consumers but also potential for greater risk taking and ill-advised consumption, rather than avoidance, of products with precautionary label statements.

The reference doses based on VITAL and proposed in this report are intended to strike a balance at a point where reactions are minimised through a combination of a high level of protection and more credible and potentially less frequent precautionary labelling. Although better data are becoming available to support the reference doses, little evidence currently exists to quantify the effect of greater credibility and more restricted use of precautionary labelling. Thus, these proposals should be seen as a starting point based on current knowledge, which will be built upon and refined as this knowledge develops.

The extent to which the proposed reference doses can be used to improve (without extending) precautionary labelling will depend to a great extent on the technology and operational characteristics of different sectors. For instance, operations where full clean-in-place (CIP) sanitation exists will generally meet the conditions more easily than those where the handling of dry materials and inaccessible machinery components preclude such techniques. In its current form, the framework only applies directly in situations where cross-contact is in non-particulate form and where the unintended allergen is mixed fairly evenly into the product. Thus, this framework may affect only a proportion of products which bear a precautionary label. However, better understanding of risks from the use of the framework in these product scenarios should also build confidence in the development of risk assessment approaches for particulate contamination scenarios as well.

\section{Risk Communication: establishing a common understanding of the risk}

Risk analysis consists of several steps of which risk assessment (science-based) and risk management (policy-based) have been discussed. Risk communication is the third major component. The Codex Alimentarius Commission Procedural Manual, Nineteenth Edition (2010) defines it as "The interactive exchange of information and opinions throughout the risk analysis process concerning risk, risk-related factors and risk perceptions, among risk assessors, risk managers, consumers, industry, the academic community and other interested parties, including the explanation of risk assessment findings and the basis of risk management decisions." This definition makes it explicit that risk communication is not a passive process of transmitting information, but an interactive and iterative one encompassing all stakeholders, including allergic consumers, where it concerns food allergens. Thus improving safety for people with food allergies requires much better communication with both allergic patients and health professionals involved in their care.

Trust in the agent delivering information lies at the heart of acceptance of any residual risk (Cvetkovich and Earle, 1992). A robust risk assessment is a necessary, but not sufficient condition for such trust. Confidence that the agent who has undertaken that risk assessment has acknowledged the interests of all those potentially affected is essential to that trust. Risk communication can be considered from a variety of perspectives, including those of the actors involved (public, private), target areas and the nature of the communication (proactive, reactive). Public risk communication concerns those areas where the public authorities are expected to take a view on, and respond to the risk, while private risk communication covers all other aspects. One example of public risk communication would be that undertaken by regulators in highlighting the standards and practices to which businesses must adhere, as well as explaining the trade-offs between different actions. Private risk communication, for example by businesses or trade associations, can also aim at similar targets, although it may arguably be less effective because of the agent delivering the message and its perceived interests. Much private risk communication will tend to be reactive, for instance in response to an incident where the safety of consumers may have been compromised. Recall notices would be a good example and need not have a wholly negative impact on trust, if well-executed. In those circumstances they can demonstrate high standards of care for customers by the affected business. Of course, any positive effect would soon evaporate and turn into reputational damage if such recall notices were to become frequent. Proactive risk communication of industry's role in driving the development of explicit defined standards for allergen management and its collaboration with other stakeholders offer a strategic chance for industry to engender more trust among consumers as appropriate active risk communication will increase trust among consumers and foster consumer loyalty.

Of course, the food industry is already communicating food (allergen) risks to consumers through mandatory declaration of priority allergens as ingredients and through precautionary labelling. However, evidence shows that communication of the risk posed by the unintended presence of allergens is largely failing, as attested by allergic consumers' understanding of, and trust in the precautionary labelling which delivers that information (Barnett et al., 2011a). In part, this lack of trust lies at the door of the best efforts of some manufacturers to be transparent about the nature of the risk their products pose. For instance, differentiated statements, such as "made on a line or in a factory that also handles allergen X" rather than simply "may contain allergen $X$ " can be an attempt to help the allergic consumer, or at least respond to demands for more meaningful information. However, since many consumers are instructed to avoid all products with precautionary allergen labelling, the large number and variety of these statements may not only create confusion among consumers but eventual distrust and even complete disregard of these statements (Cochrane et al., 2013). Disregard of precautionary statements by allergic consumers not only undermines the intent of precautionary allergen labelling but also contributes to risk-taking behaviours and potential for adverse reactions since a small but notable percentage of products with these statements contain hazardous levels of allergen (Crotty and Taylor, 2010; Hefle et al., 2007). Another factor contributing to this distrust may be the lack of common and explicit criteria used to 
apply precautionary labelling and the resulting over-use, as well as its use under circumstances where the message intrinsically lacks credibility (e.g. on bottled mineral water). This fosters, or reinforces, the belief that precautionary labelling aims more to protect the manufacturer from liability than the consumer from harm (Slovic, 1993; Slovic and MacGregor, 1994; Siegrist, 2000 2003; Renn, 1991; Lofstedt and Horlick-Jones, 1999; Leiss, 1996; Kasperson et al., 1992; Earle and Cvetkovich, 1995; Barnett et al., 2011b, 2011a; Crotty and Taylor, 2010). Restoring confidence in precautionary labelling and thereby its effectiveness will require not only explaining the benefits in terms of safety that the risk analysis framework will bring, but actively engaging all stakeholder groups in reaching agreement on the level of risk that can be accepted, as discussed in other sections. A critical aspect will be to explain how the application of such a framework to precautionary labelling will help reduce the anxiety experienced by allergic consumers and those caring for such consumers (Madsen et al., 2012).

\section{Stakeholder perspectives}

\subsection{Allergic consumers}

Communication to allergic consumers involves a high level of engagement by and with patient advocacy groups. Consumers tend to buy products on the basis of trust, experience and recommendation (Slovic, 1993; Barnett et al., 2011a). Consumers would expect that any product that is for sale is safe (subject to any exclusions for specific groups), and that safety would be assured by the producers and retailers as well as by regulatory bodies. This thinking is indeed embedded in European Food Law. All the communication to consumers via the label or package is also presumed to be part of a mandatory legal requirement. Precautionary warnings are seen by consumers as being part of this mandatory information, although in truth such warnings are voluntary and not legally regulated beyond the general requirements of food and consumer protection laws. Also, given the significant percentage of products that carry such statements [Pieretti et al., 2009], consumers are concerned that precautionary labelling is used indiscriminately, and this has undermined its value as a risk communication tool. [Hefle et al., 2007] The perception of such labels in many cases is that they do not reflect a real risk of contamination, but rather are an attempt by producers to avoid liability. Consumers appreciate industry's attempts to provide more information ("ingredients nut free", packaged in factory that uses nuts in other products" etc.), but they remain sceptical about the accuracy of these statements (Barnett et al., 2011a).

From the perspective of allergic consumers, an important consideration is that the implementation and acceptance of the proposed framework needs an appropriate communication strategy. Only then can allergic consumers be expected to understand it. A current limitation in this understanding is that most consumers do not know their individual allergen thresholds. Diagnostic testing does not generally provide this information, and there is a lack of population surveys of allergic consumers that have addressed what consumers know or understand about thresholds. Thus, consumers must rely on their personal beliefs or experiences of where their threshold lies when they encounter food products with precautionary labels. Some consumers may tend to be more cautious while others take more risks, and these tendencies likely determine their consumption behaviours. As noted previously, strict avoidance can lead to reduced food choices and nutrition for consumers, which negatively impact quality of life. On the other hand, complete disregard for precautionary labels would increase the risk to allergic cconsumers as this would contribute to consumption of foods with potentially hazardous levels of allergen (Hefle et al., 2007).
In light of consumer uncertainty about thresholds, an important feature of the new framework and indeed one of its strengths, is that the transparency of the approach lends itself well to the development of a communication strategy. Communicating a message that reference doses are associated with a certain risk of reaction provides important information about the safety of the product but also challenges the notion held by consumers that safe products are not expected to cause adverse effects under (almost) all circumstances. Thus, communication of the framework will require careful elaboration of the strategy and messages. There are precedents for such communication, for instance the microbiological risks of Listeria to certain groups, but the message here is more complex. It will require communication at different levels and cannot be achieved without full involvement of all stakeholders. There is also an important need to engage consumers about their understanding of thresholds and reference doses and about their views on risks, especially in light of potential benefits of having more products (with less precautionary labelling) available to them.

\subsection{Clinicians and other health professionals}

Health professionals such as clinicians, dieticians and nurses encounter daily the problem of how to advise patients adequately about risks from low level allergen exposures in food products. However, these advisors may not use a consistent approach in explaining risks to patients/consumers. A significant part of this problem is that there are currently no diagnostic methods available that can help health professionals accurately understand what allergen threshold doses their patients are reacting to or how severe a patient's response would be to that reactive dose. For example, prior clinical history of reaction severity has little utility in predicting which individual will have a future severe reaction (Boyce et al., 2010) and food-specific IgE levels, although higher levels predict those individuals likely to fail food challenge, do not consistently correlate with eliciting challenge doses or reaction severity (Rolinck-Werninghaus et al., 2012). Food challenges, although helpful in identifying the lowest eliciting allergen doses for a given individual, are not always performed in patients. When they are performed, clinicians are not comfortable giving patients avoidance advice based on these challenge results since data show that individual thresholds may vary over time (Leung et al., 2003a). In addition to poor diagnostic tools, health professionals are not always informed about food industry practices and thus tend to share patients' misunderstanding of the regulatory status of precautionary labelling. Anecdotally, different recommendations about avoidance of foods bearing precautionary label may be given to patients/consumers by health professionals based on personal beliefs about labelling rather than a full understanding of the risks.

Current recommended practice is to advise a given patient to avoid any exposure to the allergen, by avoiding any food containing the allergen either as an ingredient or as a possible contaminant (precautionary label statement) (Boyce et al., 2010). This approach is understandable when the intrinsic allergenicity of a food is known not to vary, e.g. peanut in loose confectionary or in wrapped cereal bars. Anecdotally, however, different avoidance advice may be given according to different clinical scenarios. For example, an adult who gets abdominal pain and minor urticaria after a full serving of well-cooked shellfish may reasonably be considered at lower risk of a subsequent episode of anaphylaxis after eating a food labelled "may contain prawn" than a peanut-allergic child with reported wheeze and collapse after consuming a tiny piece of peanut butter on bread who just ate a wrapped cereal bar labelled "may contain peanut and other nuts". Evolving clinical practice now also recognises that some allergen scenarios, such as baked egg and milk in cakes and biscuits, are tolerated and could be expected to be safe for many individuals with egg or milk allergy. In 
these cases, expert clinical advice now permits dietary introduction of milk and egg in these baked forms for appropriate individuals, while still recommending continued avoidance of all unprocessed forms of egg or milk. In practice, a dietary strategy similar to this baked food strategy could also be recommended for other allergen-containing ingredients or products should these be found to have insignificant or inconsequential levels of allergens.

Clinical advice about labelling can also be inconsistent at times. Anecdotally, some health professionals do deviate from the recommended practice and openly advocate that patients ignore all or certain precautionary labels. Consideration of analytical surveys showing high allergen levels in some labelled products suggests this to be a very risky strategy. At the same time, survey findings that many labelled products have no or very little allergen illustrate the urgent need to make precautionary labelling of prepared foods more meaningful.

A framework for the systematic and consistent application of precautionary labelling based on clear and transparent criteria clearly offers considerable scope for improving the advice provided through clinicians to allergic patients. It will enable health professionals to communicate very clearly the (low) risk implied by the absence of a precautionary label. Also, through understanding the basis of the risk assessment, they will be able to tailor their advice in a more discriminating and individualised manner to their individual patients, based on their knowledge of their allergological condition.

\subsection{Food industry}

As already discussed, the presence of unintended allergenic residues in food results from the nature of allergens as contaminants as well as from the current realities of the supply chain. Ever since allergens became a public health issue, the question "how much is too much" has been posed by food manufacturers and suppliers. Unfortunately, this question has remained largely unanswered until recently. In the meantime, manufacturers sought ways to warn allergic consumers about the potential risk from the presence of unintended allergens by the use of precautionary statements on the label, such as "may contain". While initially welcomed by allergic consumers, the spread of such statements soon reduced their credibility. Another factor which detracted from their value was the fact that different manufacturers applied different criteria to decide when to use such a label and used different phrases to convey this risk. This was perhaps not surprising since the same lack of data which drove the use of such labels also prevented the development of an agreed and consensual approach to assessing the risk from unintended allergens. Also, as mentioned, some messages from consumers indicate a desire for more differentiated information to aid them in judging risk.

While manufacturers strive to minimise cross contamination during manufacturing processes, the presence of unintended allergenic constituents by cross-contact will remain a reality for the foreseeable future because of the economic necessity to utilize shared processing facilities and lines. Consequently, precautionary labelling will remain an important risk management and risk communication tool. In this regard, the framework proposed in this report has a number of important and positive implications for all involved in the food supply chain.

Firstly, evidence-based reference doses defining a specific level of protection and providing an agreed consensual basis for the application of precautionary labelling will restore its credibility and therefore its value as a risk management and communication tool. In doing so, it will improve food safety for allergic consumers by restoring trust and communicating a clear message.

Secondly, manufacturers will benefit from a common approach to risk assessment, which can be applied right along the supply chain. This will promote greater understanding of allergen requirements between companies at different points in the supply chain including the need for quantitative information on unintended allergen content. In doing so, it will reduce the potential for misunderstandings which can lead to product recalls. A further advantage likely to flow from a set of agreed common standards is an improvement in operational capacity, leading to economies.

As alluded to earlier, however, the proposed framework also presents challenges. Achieving the standards required to avoid precautionary labelling may be quite difficult in some operations and circumstances, particularly where allergen management has had to be implemented in old facilities. As a result, some sectors will find it difficult to maintain their current level of precautionary labelling without significant investment.

Thirdly, allergen detection methods used for assessing the presence of allergen traces may find themselves at the limit of their sensitivity for some of the action levels associated with large portion sizes. There will therefore be an urgent need to be validate their limits of detection in targeted food matrixes. Accurate allergen detection methods will also be dependent on the effort of developing recognised allergen reference materials. Analytical information will then contribute to improve the accuracy of monitoring and surveillance of allergens by the food industry.

Once widely accepted, a framework of consistent standards, used by all industry and considered by regulatory authorities to be highly protective, will also remove the fear of competitive disadvantage that might otherwise hold back implementation.

From an industry point of view, thought must also be given to communicating to allergic consumers that a product has been the subject of a risk assessment and is below the action level, until such time as adoption is widespread. A reference of the type "this product has been assessed through xxx" - could make a specific reference to the proposed framework. The proposed approach will have maximum impact and therefore accrue the expected benefits to consumer safety and public health only if it is adopted widely. Clearly this will require significant education and training, with a particular focus on the very numerous medium and small companies who may find it difficult to access the relevant technical expertise. The example of the implementation of VITAL in Australia will provide useful experience in this regard.

\subsection{Public health authorities}

The development and implementation of a framework for allergen risk assessment anchored in evidence-based reference doses will give public health authorities, including regulators and enforcement bodies, a more secure basis for enforcement activities when allergen cross contamination is detected in a product, thereby providing greater clarity and more consistency for businesses in the handling of food allergen incidents. In this regard, it may provide a basis for assessing overall population health hazard associated with certain allergen exposures in contaminated or recalled products and be used to improve guidance about these hazards to industry as well as allergic consumers and healthcare practitioners. This risk assessment framework may also be a tool to provide scientific evidence to support exemption claims from source labelling for ingredients demonstrated to contain allergen content and expected product exposures well below those described by reference dose limits.

\section{Accepted level of risk}

Widespread use of precautionary labelling could be viewed as an attempt to prevent any and all types of reaction in every allergic individual, i.e. trying to achieve zero risk, which is not a realistic possibility. The severity of adverse events varies significantly between persons with a food allergy and depends on the nature and 
properties of the allergen, the amount of the allergen consumed and the physiological state and genetic background of the patient. A prerequisite for public health authorities is therefore to share a broadly similar outlook on the frequency of food allergic reactions that could be accepted, differentiating between reactions of different degrees of severity and aligned with the actual needs and behaviour of allergic consumers. With regard to products with precautionary label statements, some degree of reaction risk from consumption of these products may be more acceptable from a public health standpoint if it can be demonstrated that current or potential risks (e.g. negative quality of life, adverse reactions from risk-taking behaviours, etc.) could be lessened. Thus, the protection of allergic consumers is a shared responsibility and regulators across the EU and beyond should make that decision in consultation with all interested parties, including allergic consumers, patient organisations, food businesses, scientists and healthcare professionals (i.e. medical doctors, health centre physicians and dieticians) and public authorities. Agreement between stakeholders on what can be achieved and the resulting risk management objectives would form a sound basis for progress, building on existing evidence about risk perception and behaviours (Madsen et al., 2012; Health Council of the Netherlands, 2007). Once a political decision has been made on the tolerable frequency of different types of adverse allergic effects, as discussed above, allergen reference doses can be determined which meet the appropriate level of protection and can be used by businesses and regulators to assess the levels of allergen cross contamination that have been detected in a particular product. If a business is able to ensure that this Reference Dose would not be exceeded by estimated per meal consumption of the allergen-containing food product in question, the precautionary labelling should be omitted on that particular product (Health Council of the Netherlands, 2007). Implementation of such an approach is supported by the observation that, as discussed in more detail in an accompanying paper (ref), any given benchmark, such as a Reference Dose, actually protects to a greater degree than the nominal level of protection, because of the way the benchmark is interpreted and implemented to assure compliance. Thus, if a business is controlling allergen cross contamination to a certain level, almost all products will contain allergen below that level, if any at all. Products that contain allergen at the action level would be in the minority.

\section{How would risk managers apply these values in different scenarios?}

The effectiveness with which new standards can be applied, monitored by businesses and enforced by authorities will be critical to their adoption and use. As already discussed, precautionary allergen labelling has lost credibility and effectiveness (Barnett et al., 2011b). The standards aim not only to harmonise the implementation of precautionary labelling across industry, but to limit (or at least not increase) its extent. It will therefore be very important to strike the correct balance between ensuring a sufficient range of food choices to limit risk-taking by allergic consumers and yet minimising reactions due to unintended allergen presence below the reference dose. Setting reference doses also needs to consider the adequacy of analytical techniques to detect the allergens reliably at the concentrations that would be found in products if unintended allergen was present around the action level.

Finally, it is paramount that any set of allergen reference doses that are established are understood by consumers and the health care community and can be used by them without reservations to make informed choices. The basis for the reference doses selected and the data underpinning those values need to be clear, as do any assumptions that are made. The risk/benefit balance is between protecting the allergic consumer as much as is practicable, whilst enabling businesses to produce products that are economically viable and that do not result in unnecessary restrictions in consumer choice and quality of life. This needs to be explained to the consumer and to health professionals, and the improvements in comparison with the current situation set out.

\section{Future perspectives}

\subsection{Global}

Food allergy is of global and growing importance to public health, affecting consumers' quality of life (mainly children) and making increasing demands on health service resources. Developing knowledge about the relationship between allergen dose and population reactivity, as well as the tools to translate this knowledge to practical action to improve safety and quality of life of allergic consumers bring the possibility to manage food allergens as effectively as other food safety hazards.

Optimal protection of allergic consumers against allergen risks rests on the application of risk management practices based on scientifically robust principles, shared across all stakeholders. This is equally valid when prioritising food allergens on the basis of their public health impact, as in the lists of regulated allergens.

Allergen labelling represents a critical tool for management and needs to communicate the allergen status of products unambiguously using simple, harmonized terms in local language to maximise consumer understanding available upon demand. Precautionary warnings must be applied consistently for unintended allergen presence using quantitative action levels and using harmonised phrases to indicate such risks to assure integrity and retain relevance.

The global nature of today's food supply chain dictates that effective management depends on application of good practice across all jurisdictions worldwide by all stakeholders i.e. regulators, food manufacturers, retailers and caterers alike. Enshrining these principles in a CODEX code of practice for the risk management of food allergens that would reach out globally and describe a consistent standard approach could be a possible way to achieve this goal.

\subsection{Promoting positive distinction}

There is concern that allergen precautionary warnings are currently so devalued that they are ignored by a significant proportion of food-allergic consumers and some health professionals do not contradict their patients when they ignore such warnings. A current barrier to effectively protecting allergic consumers is their inevitable lack of understanding of the meaning of precautionary labelling in the context of the disparate standards. However moving to the harmonised system discussed in this series of papers will require clear communication of the changes to those consumers, as well as to health professionals. In particular, while the use of precautionary warning labels remains voluntary, allergic consumers may want to know whether a product that bears no warning does so because a risk assessment referenced to accepted standards was conducted and found no need for this label warning. Two different messages concerning the interpretation of labelling could be construed; these are:

- A risk assessment has been conducted using a specified system based on agreed allergen reference doses, as a result of which a precautionary warning is warranted. The precautionary statement would also need to be limited to one or two phrases, whose use would be restricted to companies using the system.

- A risk assessment has been conducted and a decision taken that the risk is negligible, together with a positive message to indicate this. Such a positive message could provide a 
useful incentive for companies to adopt the new framework, effectively providing a "dividend" for the investment in learning about it and implementing it.

Consumers with food allergies could then actively seek out the 2nd category of products, while understanding that the absence of a warning does not guarantee that there is zero cross contamination risk. They should therefore have been advised by their healthcare practitioners that their allergy was such that the risk to them was indeed negligible. There could also be a third category of products without any messages beyond mandatory labelling of regulated allergens in which it is implied that the business has not conducted a risk assessment of the appropriate rigour.

\subsection{Need for frequent revisions}

Allergen reference values developed as part of the work of this group are based on varying levels of data. Whilst the numbers of challenges in subjects with allergies to peanut, egg, milk and hazelnut appear to be sufficient to produce robust outcomes, this is not so for some of the other major food allergens covered by the regulatory allergen labelling list in Europe and for some the data are currently very sparse. Moreover, quality assessments of the available challenge data and models used in the determination of the reference doses need to be conducted. As challenge data continue to be produced for those food allergens for which some data exist but are not yet fully sufficient to derive robust outcomes, the provisional reference values currently put forward will need to be reviewed at suitable intervals, until the outcomes are considered to be robust. Where the data currently available are not sufficient to derive even provisional reference values, consideration should be given to the possibility of using a 'default' reference value by extrapolating from those food allergens where robust reference values have been derived.

\section{Conflict of Interest}

A. Baka is employed by ILSI Europe.

S. Luccioli did not receive funding from ILSI Europe and the findings and conclusions in this article should not be construed to represent US FDA determination or policy.

S. Hattersley is a food allergy expert employed by the UK Food Standards Agency and was invited to take part in this expert group. She received no ILSI funding for this participation.

J.O'B. Hourihane has received research funding from Danone, the UK Food Standards Agency and the Food Allergy Research and Resource Program at the University of Nebraska, USA and has received speaker fees from Danone/Nutricia and Mead Johnson.

\section{Transparency Document}

The Transparency document associated with this article can be found in the online version.

\section{Acknowledgements}

This work was conducted by an expert group of the European branch of the International Life Sciences Institute (ILSI Europe). The expert group received funding from the ILSI Europe Food Allergy Task Force. Industry members of this task force are listed on the ILSI Europe website at www.ilsi.eu. For further information about ILSI Europe, please email info@ilsieurope.be or call +32 2771 00 14. The opinions expressed herein and the conclusions of this publication are those of the authors and do not necessarily represent the views of ILSI Europe nor those of its member companies.

The authors wish to thank the members of the Expert Group: Dr. Anke Ehlers, Dr. Marcel Feys, Dr. Corinne Herouet-Guicheney, Dr. Geert Houben, Dr. André Knulst, Prof. Marek Kowalski, Prof. Alfonso Lampen, Dr. Charlotte Madsen, Dr. Hubert Noteborn, Prof. Nikolaos Papadopoulos, Dr. Fabrice Peladan, Prof. Stephen Taylor, and Prof. Margitta Worm for their invaluable contribution to this work through their enthusiastic and generous participation.

\section{Appendix A.}

Annex

Glossary for Expert Group

Term

Hazard

\section{Definition}

sys

Inherent property of a substance or situation having the potential to cause an adverse effect to an organism,

Exposure $\quad$ Concentration or amount of a particular agent that reaches a target organism, system, or (sub)population in a specific frequency for a defined duration

Routes of exposure Different modes of entry into the organism after contact, for example dermal, ingestion, inhalation

Intake

Risk

Safety

Risk factors Amount of exposure to a substance as part of food and/or water consumed Probability of an adverse effect in an organism, system or (sub)population caused under specified circumstances of exposure to a substance or situation Practical certainty that adverse effect swill not arise upon exposure to a substance under defined exposure circumstances

Any characteristic of the patient, environment or food which can alter the likelihood or severity of an adverse reaction

Host factors Factors pertaining specifically to the host or patient, such as genetics, medical conditions, etc.

Event factors Factors relating to the event or exposure occasion, such as place, circumstances etc

Assessment factor Numerical adjustment used to extrapolate from experimentally determined (dose-response) relationships to estimate the agent exposure below which an adverse effect is not likely to occur

Uncertainty factor Reductive factor by which an observed or estimated no-observed-adverse effect level (NOAEL) is divided to arrive at a criterion or standard that is considered safe or without appreciable risk

Related terms: Assessment factor, Safety factor

Safety factor Composite (reductive) factor by which an observed or estimated no-observed 
LOAEL

LOEL

NOAEL

NOEL

Benchmark dose

Eliciting dose

Minimum eliciting dose (MED)

Minimum provoking dose (MPD)

Dose distribution

Dose

Reference dose

Guidance value

Threshold adverse-effect level (NOAEL) is divided to arrive at a criterion or standard that is considered safe or without appreciable risk Related terms: Assessment factor, Uncertainty factor

Lowest Observed Adverse Effect Level in a controlled experimental study the lowest dose which results in an adverse effect

Lowest Observed Effect Level - in a controlled experimental study the lowest dose which results in any observable effect No Observed Adverse Effect Level - in a controlled experimental study the highest dose which does not result in an adverse effect

No Observed Effect Level - in a controlled experimental study the highest dose which does not result in any observable effect

A dose of an allergenic food that when consumed produces a reaction in a predetermined proportion of the susceptible (allergic) population The dose in a dose distribution which is predicted to provoke reactions in a defined proportion of allergic individuals (denoted as Ed p where $\mathrm{p}$ is the proportion)

The minimum dose that elicits an effect in an individual in a challenge study equivalent to an individual's LOAEL Synonym of MED

The plot describing the cumulative proportion of (allergic) individuals reacting as a function of dose, based on their MEDs

Total amount of an agent administered to, taken up by, or absorbed by an organism, system, or (sub)population An estimate of the daily exposure dose that is likely to be without deleterious effect even if continued exposure occurs over a lifetime. In the case of allergens, since acute exposure defines risk for adverse deleterious effect, the exposure estimate is derived from amount per eating occasion

Related term: Acceptable daily intake Value, such as concentration in air or water, that is derived after allocation of the reference dose among the different possible media (routes) of exposure The aim of the guidance value is to provide quantitative information from risk assessment to the risk managers to enable them to make decisions (See also Reference dose) Dose or exposure concentration of an agent below which a stated effect is not observed or expected to occur It lies in an interval bounded by the LOAEL (upper) and NOAEL (lower)

Action level The concentration of an allergen in a product below which a precautionary label is deemed unnecessary (derived from reference value)

Precautionary label An advisory statement or warning for consumers accompanying, or available at point of sale of, foodstuffs which provides risk communication to subpopulations with special dietary requirements, e.g. phenylalanine intolerance, lactose intolerance, milk allergy

\section{References}

Allen, K.J., Remington, B.C., Baumert, J.L., Crevel, R.W., Houben, G.F., Brooke-Taylor S., Kruizinga, A.G., Taylor, S.L., 2013. Allergen reference doses for precautionary labeling (VITAL 2.0): clinical implications. J. Allergy Clin. Immunol..

Allergen Bureau, 2012 Voluntary Incidental Trace Allergen Labelling (VITAL)

Barnett, J., Leftwich, J., Muncer, K., Grimshaw, K., Shepherd, R., Raats, M.M. Gowland, M.H., Lucas, J.S., 2011a. How do peanut and nut-allergic consumers use information on the packaging to avoid allergens? Allergy 66, 969-978.

Barnett, J., Muncer, K., Leftwich, J., Shepherd, R., Raats, M.M., Gowland, M.H. Grimshaw, K., Lucas, J.S., 2011b. Using 'may contain' labelling to inform food choice: a qualitative study of nut allergic consumers. BMC Public Health 11 734.

Boyce, J.A., Assa’ad, A., Burks, A.W., Jones, S.M., Sampson, H.A., Wood, R.A., Plaut, M., Cooper, S.F., Fenton, M.J., Arshad, S.H., Bahna, S.L., Beck, L.A., Byrd-Bredbenner, C., Camargo Jr., C.A., Eichenfield, L., Furuta, G.T., Hanifin, J.M., Jones, C., Kraft, M. Levy, B.D., Lieberman, P., Luccioli, S., McCall, K.M., Schneider, L.C., Simon, R.A. Simons, F.E., Teach, S.J., Yawn, B.P., Schwaninger, J.M., 2010. Guidelines for the diagnosis and management of food allergy in the United States: report of the NIAID-sponsored expert panel. J. Allergy Clin. Immunol. 126, S1-S58.

Christie, L., Hine, R.J., Parker, J.G., Burks, W., 2002. Food allergies in children affect nutrient intake and growth. J. Am. Diet. Assoc. 102, 1648-1651.

Cochrane, S.A., Gowland, M.H., Sheffield, D., Crevel, R.W.R., 2013. Characteristics and purchasing behaviours of food-allergic consumers and those who buy food for them in Great Britain. Clin. Transl. Allergy 3, 31.

Crotty, M.P., Taylor, S.L., 2010. Risks associated with foods having advisory milk labeling. J Allergy Clin. Immunol. 125, 935-937.

Cvetkovich, G., Earle, T., 1992. Environmental hazards and the public. J. Social Issues $48,1-20$.

Earle, T., Cvetkovich, G., 1995. Social Trust: Toward a Cosmopolitan SocietyPraeger, Westport, CT.

Federal Register, 2013 Food Labeling; Gluten-Free Labeling of Foods. 21 CFR part 101 ed.

Health Council of the Netherlands, 2007 Food allergy. Publication no. 2007/07 ed. The Hague.

Hefle, S.L., Furlong, T.J., Niemann, L., Lemon-Mule, H., Sicherer, S., Taylor, S.L., 2007 Consumer attitudes and risks associated with packaged foods having advisory labeling regarding the presence of peanuts. J Allergy Clin. Immunol. 120, 171176.

Kasperson, R.E.D., Golding, D., Tuler, S., 1992. Siting hazardous facilities and communicating risks under conditions of high social distrust. J. Social Issues 48 , $161-172$.

Leiss, W., 1996. Three phases in the evolution of risk communication practice. Ann. Am. Acad. Political Social Sci. 545, 85-94.

Leung, D.Y., Sampson, H.A., Yunginger, J.W., Burks Jr., A.W., Schneider, L.C., Wortel, C.H., Davis, F.M., Hyun, J.D., Shanahan Jr., W.R., 2003a. Effect of anti-IgE therapy in patients with peanut allergy. N. Engl. J. Med. 348, 986-993.

Leung, D.Y., Shanahan Jr., W.R., Sampson, H.A., 2003b. Response to "effect of anti-IgE therapy in patients with food allergy". Ann. Allergy Asthma Immunol. 91, 515517.

Lofstedt, R.E., Horlick-Jones, T., 1999. Environmental regulation in the UK: politics, institutional change and public trust. In: Cvetkovich, G., Lofstedt, R.E. (Eds.) Social Trust and the Management of Risk Earthscan, London.

Madsen, C.B., Crevel, R., Chan, C.H., Dubois, A.E., DunnGalvin, A., Flokstra-de Blok, B.M., Gowland, M.H., Hattersley, S., Hourihane, J.O., Norhede, P., Pfaff, S., Rowe G., Schnadt, S., Vlieg-Boerstra, B.J., 2010. Food allergy: stakeholder perspectives on acceptable risk. Regul. Toxicol. Pharmacol. 57, 256-265.

Madsen, C.B., Hattersley, S., Allen, K.J., Beyer, K., Chan, C.H., Godefroy, S.B., Hodgson, R., Mills, E.N., Munoz-Furlong, A., Schnadt, S., Ward, R., Wickman, M., Crevel, R. 2012. Can we define a tolerable level of risk in food allergy? Report from a EuroPrevall/UK Food Standards Agency workshop. Clin Exp. Allergy 42, 30-37. 
Pieretti, M.M., Chung, D., Pacenza, R., Slotkin, T., Sicherer, S.H., 2009. Audit of manufactured products: use of allergen advisory labels and identification of labeling ambiguities. J. Allergy Clin. Immunol. 124, 337-341.

Rance, F., Grandmottet, X., Grandjean, H., 2005. Prevalence and main characteristics of schoolchildren diagnosed with food allergies in France. Clin. Exp. Allergy 35, $167-172$.

Renn, O., 1991. Risk communication and the social amplification of risk. In: Kasperson, R.E.D., Stallen, P.J. (Eds.), Communicating Risks to the Public: International Perspectives. Kluwer, Dordrecht.

Rimbaud, L., Heraud, F., La Vieille, S., Leblanc, J.C., Crepet, A., 2010. Quantitative risk assessment relating to adventitious presence of allergens in food: a probabilistic model applied to peanut in chocolate. Risk Anal. 30, 7-19.

Rolinck-Werninghaus, C., Niggemann, B., Grabenhenrich, L., Wahn, U., Beyer, K., 2012. Outcome of oral food challenges in children in relation to symptomeliciting allergen dose and allergen-specific IgE. Allergy 67, 951-957.

Siegrist, M., 2000. The influence of trust and perceptions of risks and benefits on the acceptance of gene technology. Risk Anal. 20, 195-203.
Siegrist, M., 2003. Perception of gene technology and food risks: results of a survey in Switzerland. J. Risk Res. 6, 45-60.

Slovic, P., 1993. Perceived risk, trust, and democracy. Risk Anal. 13, 675-682.

Slovic, P., MacGregor, D., 1994. The Social Context of Risk Communication Decision Research, Eugene, Oregon.

Taylor, S.L., Baumert, J.L., Kruizinga, A.G., Remington, B.C., Crevel, R.W., BrookeTaylor, S., Allen, K.J., Houben, G., 2014. Establishment of reference doses for residues of allergenic foods: report of the VITAL expert panel. Food Chem. Toxicol. 63, 9-17.

Wainstein, B.K., Studdert, J., Ziegler, M., Ziegler, J.B., 2010. Prediction of anaphylaxis during peanut food challenge: usefulness of the peanut skin prick test (SPT) and specific IgE level. Pediatr. Allergy Immunol. 21, 603-611.

Zurzolo, G.A., Allen, K.J., Taylor, S.L., Shreffler, W.G., Baumert, J.L., Tang, M.L.K., Gurrin, L.C., Mathai, M.L., Nordlee, J.A., DunnGalvin, A., Hourihane, J. O’B, 2013. Allergy Asthma Clin. Immunol. 9, 35, <http://www.aacijournal.com/content/9/ $1 / 35>$. 